\title{
Mental health problems of children in the community: 18 month follow up
}

\author{
Robert Goodman, Tamsin Ford, Howard Meltzer
}

Department of Child and Adolescent

Psychiatry, Institute of Psychiatry, King's College London, London SE5 8AF Robert Goodman professor

Tamsin Ford clinical research fellow Office for National Statistics, London SW1V 2QQ

Howard Meltzer principal social researcher

Correspondence to: Robert Goodman r.goodman@iop. kcl.ac.uk

BMJ 2002;324:1496-7

In a recent national survey, the prevalence of psychiatric disorder in children and adolescents in Great Britain was more than $9 \% .{ }^{1}$ Parents and doctors commonly think that these disorders are transient, but longitudinal studies show otherwise. ${ }^{2}$ We followed up children from the national survey to examine persistence in a large sample of children in Britain. ${ }^{1}$

\section{Participants, method, and results}

The original survey studied a sample of 10438 British children aged 5-15 years. ${ }^{1}$ We sent the parents of all children with a psychiatric disorder (936) and a third of those without a psychiatric disorder (3029) a questionnaire. It included the strengths and difficulties questionnaire, a well validated measure of childhood psychopathology and its impact on the child. ${ }^{3}$

A total of 73\% of parents responded; most losses were non-contacts, not refusals. The response rate was lower among those with a child with a disorder than those without $(66 \%$ v 76\%). Non-responders at follow up had had significantly more symptoms initially (analysis of variance adjusting for initial diagnosis, $\mathrm{F}=39.9,1 \mathrm{df}, \mathrm{P}<0.001$ : the mean symptom score for non-responders was greater, by 1.2 , than for responders, matched by diagnosis). Responders' and nonresponders' initial impact scores did not differ significantly. Overall, differential non-response will not have led us to overestimate the persistence of psychopathology.

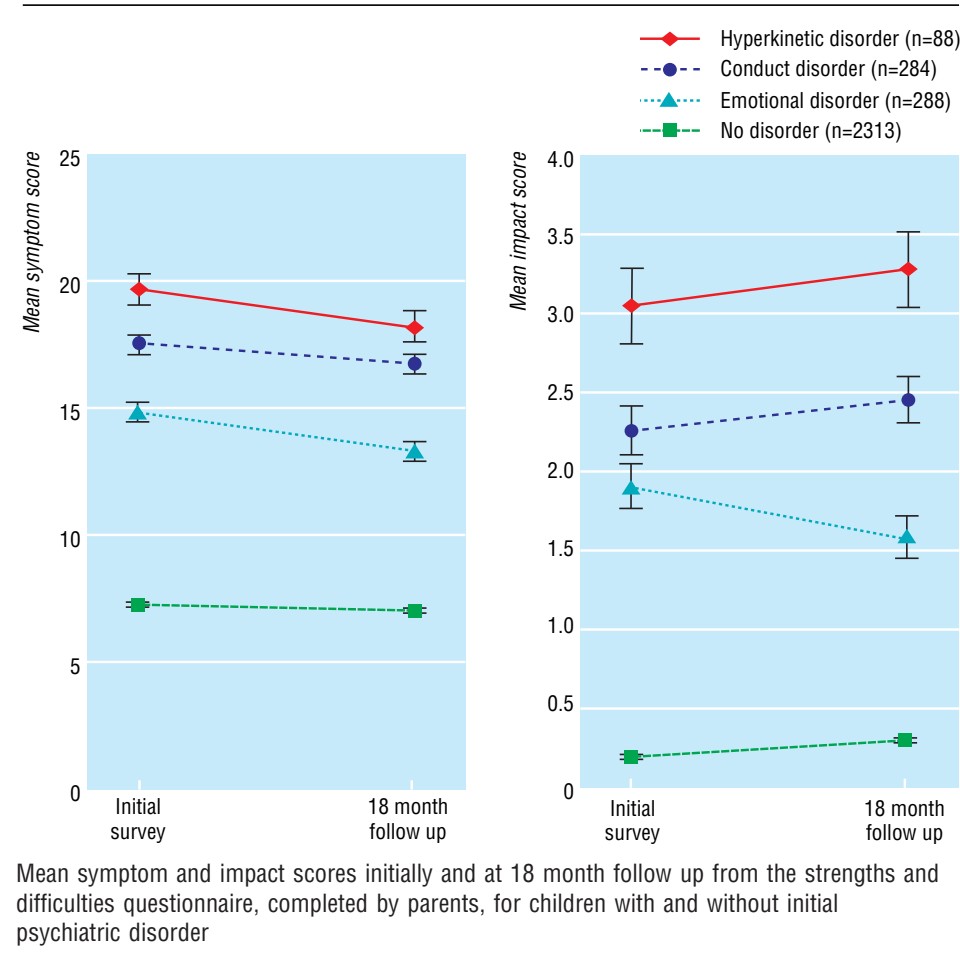

difficulties questionnaire, completed by parents, for children with and without initial (nisorder
The figure shows symptom and impact scores in children initially diagnosed with hyperkinetic, conduct, emotional, or no disorder. We used paired $t$ tests to test whether the changes in symptom and impact scores in children with hyperkinetic, conduct, emotional, or no disorder were significant. For conduct disorder, neither symptoms nor impact changed significantly. Symptoms $(\mathrm{P}<0.05)$ but not impact fell significantly in children with hyperkinesis. For emotional disorder, there were significant falls in both symptoms and impact ( $\mathrm{P}<0.001$ for both), though the follow up scores were still substantially greater than in the group with no initial disorder $(\mathrm{P}<0.001$, independent samples $t$ test).

We defined "caseness" from the combination of raised symptom $(\geqslant 14)$ and impact scores $(\geqslant 2){ }^{3}$ Caseness at follow up was considered informative for only the $86 \%(2487 / 2901)$ of participants in whom caseness was congruent with the initial psychiatric diagnosis. For example, when a child was initially diagnosed as having conduct disorder solely on the basis of symptoms reported by a teacher, and when the initial questionnaire, completed by the parents, described their child as normal, then the diagnosis (disorder present) and the caseness (negative) were not congruent. So, caseness was not considered informative at follow up, since the parent's answers to the questionnaire would probably not change whether or not the conduct disorder persisted or was resolved. Similarly, if the initial diagnosis of depression in a teenager was based on self reported symptoms (not reported by a parent), the follow up parent questionnaire is unlikely to be informative.

Caseness criteria at the 18 month follow up were met by $3 \%(67 / 2249)$ of children who did not initially have a psychiatric disorder, compared with $62 \%$ (147/ 238) of children who did (relative risk 20.7; $95 \%$ confidence interval 16.0 to 26.7). Persistence at 18 months varied with initial diagnosis: $36 \%(25 / 70)$ for children who had an emotional disorder initially, compared with 73\% (122/168) for children with other disorders initially (mostly conduct and hyperkinetic disorders, sometimes with coexistent emotional disorders) $\left(\mathrm{P}<0.001, \chi^{2}\right.$ test $)$.

\section{Comment}

Childhood psychopathology is often persistent, particularly among children with conduct disorder and hyperkinesis. Although emotional disorders have a better prognosis than conduct or hyperkinetic disorders, they are not always benign: their resolution over 18 months is far from complete, and recent work suggests an increased risk of similar disorders recurring in adulthood. ${ }^{2}$

Everyone in contact with children should take the symptoms of emotional distress, behavioural difficulty, and hyperactivity seriously, as they may impair the child's function and development and are unlikely to be transient. This is particularly important as evidence 
based interventions can alleviate distress and minimise the secondary handicap that results from disrupted education and impaired social development. ${ }^{5}$

Contributors: HM directed the initial survey and the follow up and discussed the analyses, findings, and draft versions of the paper. RG and TF were on the steering committee for both surveys. RG performed the analyses while TF took the lead in drafting the paper. RG is guarantor.

Funding. TF is funded by a Wellcome clinical training fellowship and the original surveys were funded by the Department of Health.
Competing interests: None declared.

1 Meltzer H, Gatward R, Goodman R, Ford T. Mental health of children and adolescents in Great Britain. Stationery Office: London, 2000.

2 Harrington R. Developmental continuities and discontinuities. $\mathrm{Br} J$ Psychiatry 2001;179:189-90.

3 Goodman R. The extended version of the strengths and difficulties questionnaire as a guide to child psychiatric caseness and consequent burden. J Child Psychol Psychiatry 1999;40:791-801.

4 Goodman R. Psychometric properties of the strengths and difficulties questionnaire (SDQ). J Am Acad Child Adolesc Psychiatry 2001;40:1337-45.

5 Graham P. Treatment interventions and findings from research: bridging the chasm in child psychiatry. Br J Psychiatry 2000;176:414-9.

(Accepted 27 February 2002)

\section{Drug points}

\section{Severe myalgia from an interaction between} treatments with pantoprazole and methotrexate

U Tröger, Institute of Clinical Pharmacology, B Stötzel, Department of Dermatology and Venerology, J Martens-Lobenhoffer, Institute of Clinical Pharmacology, H Gollnick, Department of Dermatology and Venerology, F P Meyer, Institute of Clinical Pharmacology, Hospital of the Otto-von-Guericke-University, D-39120 Magdeburg, Germany

A 59 year old man with a folliculotropic cutaneous $\mathrm{T}$ cell lymphoma had been receiving treatment with interferon alfa-2a. After the treatment was discontinued because his tumour had relapsed, he started taking low dose pulse methotrexate (15 mg intramuscularly, once a week). Concomitantly he also had a Barrett oesophagus, which was treated with pantoprazole (20 $\mathrm{mg}$ /day, orally), and arterial hypertension, which was treated with atenolol. After the first injection of methotrexate the patient had severe generalised myalgia and bone pain. This led to partial immobility that began three to four hours after the injection and continued-albeit to a lesser degree-for several days. The symptoms recurred over the following four methotrexate cycles. Multiple clinical and laboratory examinations excluded clinical causes such as an underlying focal or disseminated infection and the systemic exacerbation of the cutaneous $\mathrm{T}$ cell lymphoma. A drug interaction with methotrexate was suspected because the symptoms arose when the drug was given. After pantoprazole was replaced by ranitidine, the symptoms subsided dramatically and finally disappeared.

The patient was rechallenged with pantoprazole eight weeks later, having given his informed consent. Treatment with pantoprazole was started six days before the weekly dose of methotrexate was given so that the maximum effect could be attained. Serum concentrations of methotrexate and its metabolite 7-hydroxymethotrexate were monitored with and without pantoprazole by a high performance liquid chromatography-fluorescence assay. The two study periods were separated by a washout phase of six weeks. Atenolol, clemastine to treat local itching, and ascorbic acid to treat a temporary deficiency of vitamin C were given concomitantly.

The symptoms reappeared in response to the challenge but did not reappear without pantoprazole. The concentration-time curves (figure) are identical for methotrexate in both periods, but they differ considerably for 7-hydroxymethotrexate. The area under the curve between 0 and $144 \mathrm{~h}$ after drug administration was nearly $70 \%$ higher for 7-hydroxymethotrexate plus pantoprazole than without pantoprazole (1929 $\mathrm{ng} \cdot \mathrm{h} / \mathrm{ml} \quad v \quad 1131$ $\mathrm{ng} \cdot \mathrm{h} / \mathrm{ml}$ ); the half life of 7-hydroxymethotrexate was doubled when methotrexate was given with pantoprazole $(81.4 \mathrm{~h} v 36.4 \mathrm{~h})$. This indicates an interaction in renal elimination, rather than a metabolic interaction.

About $30 \%$ of all patients discontinue the low dose pulse methotrexate because of adverse effects. Drug interactions contribute considerably to the number of patients who stop using the drug. ${ }^{2}{ }^{3}$ Reactions after dosing occur in
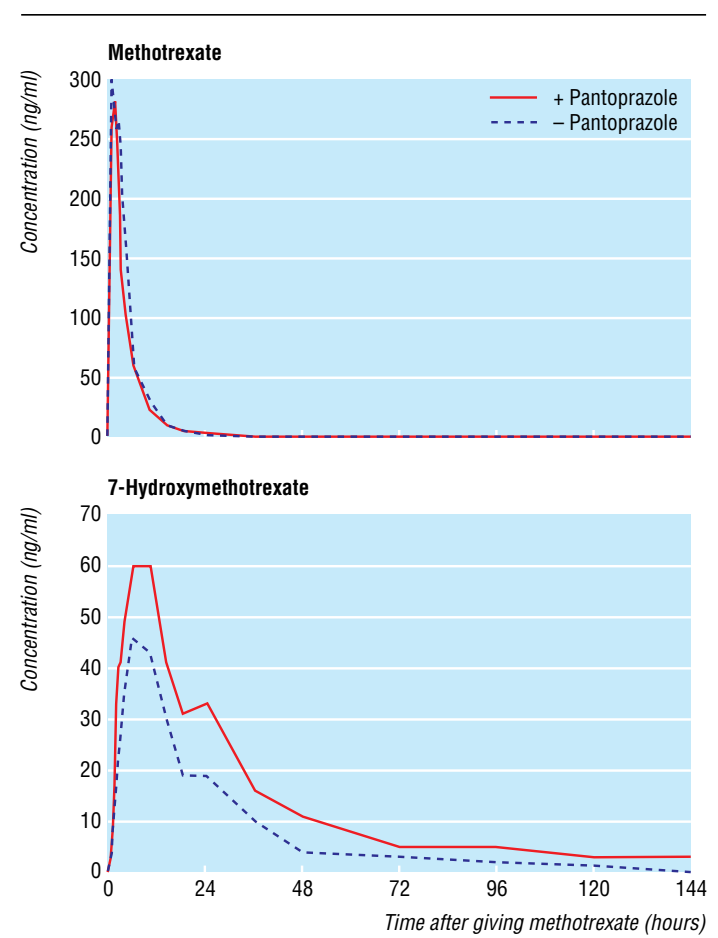

Serum concentration of methotrexate and 7-hydroxymethotrexate with and without pantoprazole

about $10-15 \%$ of patients with rheumatoid arthritis receiving this treatment. ${ }^{45}$ This report shows for the first time a link between methotrexate post-dosing reactions and an interaction of 7-hydroxymethotrexate and concomitantly given pantoprazole. The German Federal Institute for Drugs and Medical Devices and the Drug Commission of the German Medical Profession were informed about the case. The manufacturer of methotrexate (Medac $\mathrm{GmbH}$, Wedel, Germany) was not aware of this drug interaction.

Funding: None. Competing interests: None declared.

1 Beck O, Seideman P, Wennberg M, Peterson C. Trace analysis of methotrexate and 7-hydroxymethotrexate in human plasma and urine by a novel high-performance liquid chromatographic method. Ther Drug Monit 1991;13:528-32

2 Van Dooren-Greebe RJ, Kuijpers AL, Termorshuizen F, van de Kerkho PC. Interruption of long-term methotrexate treatment in psoriasis. Evaluation of clinical course and laboratory parameters after discontinution and reintroduction of weekly oral methotexate Ada Dem Vered :393-6.

3 van Ede AE, Laan RFJM, Blom HJ, De Abreu RA, van de Putte LBA. Methotrexate in rheumatoid arthritis: an update with focus on mechanisms involved in toxicity. Semin Arthritis Rheum 1998;27:277-92.

4 Halla JT, Hardin JG. Underrecognized postdosing reactions to methotrexate in patients with rheumatoid arthritis. J Rheumatol 1994;21:1224-6.

5 McKendry RJ. The remarkable spectrum of methotrexate toxicities Rheum Dis Clin North Am 1997;23:939-54. 